\title{
Erratum: Proximity effect in superconducting heterostructures with strong spin-orbit coupling and spin splitting [Phys. Rev. B 100, 104514 (2019)]
}

\author{
Yao Lu and Tero T. Heikkilä
}

(Received 16 December 2019; published 21 January 2020)

DOI: 10.1103/PhysRevB.101.049908

There are some errors in this paper. The conclusions are not affected by these errors, but the following corrections should be made. The $p_{F}$ in the first term of Eq. (7) should be a vector $\boldsymbol{p}_{\boldsymbol{F}}$. The two terms $i \boldsymbol{h}^{\prime} \cdot \sigma^{\prime}+i h_{z} \sigma_{3}$ on the right-hand side of Eq. (7) should be $i \boldsymbol{h}^{\prime} \cdot \sigma^{\prime} \tau_{3}+i h_{z} \sigma_{3} \tau_{3}$. We change Eq. (7) to be

$$
\frac{\boldsymbol{p}_{\boldsymbol{F}}}{m_{N}} \nabla \hat{g}+\frac{\alpha}{2}\{\boldsymbol{\eta}, \nabla \hat{g}\}=\left[\hat{g}, \omega_{n} \tau_{3}+i \boldsymbol{h}^{\prime} \cdot \sigma^{\prime} \tau_{3}+i h_{z} \sigma_{3} \tau_{3}+i \alpha p_{F} \boldsymbol{\eta} \cdot \boldsymbol{n}_{F}+\frac{\langle\hat{g}\rangle}{2 \tau}\right] .
$$

Below Eq. (8), the definition of the $\hat{Q}$ operator should be $\hat{Q} \equiv i h_{z} \sigma_{3} \tau_{3}+i \alpha p_{F} \boldsymbol{\eta} \cdot \boldsymbol{n}_{F}$. Similar typographical errors appear in Eq. (13), and we change Eq. (13) to be

$$
v_{F} \boldsymbol{n}_{F} \cdot \nabla \hat{g}=\left[\hat{g}, \omega_{n} \tilde{\tau}_{3}+i \frac{\alpha p_{F}}{S_{F}}\left(\boldsymbol{h}^{\prime} \times \hat{z}\right) \cdot \boldsymbol{n}_{F} \tilde{\tau}_{3}+\frac{\langle\hat{g}\rangle}{2 \tau}\right] .
$$

Here, we take the opportunity to provide the derivation of Eq. (13). Let us start with the Gorkov equation,

$$
\left(\hat{G}_{0}^{-1}+\mu-\hat{\Sigma}\right) \otimes \hat{G}\left(\boldsymbol{r}_{1}, \boldsymbol{r}_{2}\right)=\delta\left(\boldsymbol{r}_{1}-\boldsymbol{r}_{2}\right),
$$

with

$$
\hat{G}_{0}^{-1}=-\frac{\hat{\boldsymbol{k}}^{2}}{2 m_{N}}-\alpha \hat{\boldsymbol{k}} \cdot \boldsymbol{\eta}+\left(i \omega_{n}+\boldsymbol{h} \cdot \boldsymbol{\sigma}\right) \tau_{3} .
$$

Here, the convolution operator $\otimes$ is defined by

$$
A\left(\boldsymbol{r}_{1}, \boldsymbol{r}_{3}\right) \otimes B\left(\boldsymbol{r}_{3}, \boldsymbol{r}_{2}\right) \equiv \int d \boldsymbol{r}_{3} A\left(\boldsymbol{r}_{1}, \boldsymbol{r}_{3}\right) B\left(\boldsymbol{r}_{3}, \boldsymbol{r}_{2}\right) .
$$

We define the "center-of-mass coordinate" $\boldsymbol{R}=\frac{\boldsymbol{r}_{1}+\boldsymbol{r}_{2}}{2}$ and the coordinate difference $\boldsymbol{r}=\boldsymbol{r}_{1}-\boldsymbol{r}_{2}$. We perform Fourier transformation with respect to $r$ for all the operators in Eq. (S1) according to

$$
A(\boldsymbol{p}, \boldsymbol{R})=\int d \boldsymbol{r} A\left(\boldsymbol{R}+\frac{1}{2} \boldsymbol{r}, \boldsymbol{R}-\frac{1}{2} \boldsymbol{r}\right) e^{-i \boldsymbol{p} \cdot \boldsymbol{r}},
$$

and perform a gradient expansion,

$$
(A \otimes B)(\boldsymbol{p}, \boldsymbol{R}) \approx\left(1+\frac{i}{2} \partial_{\boldsymbol{R}}^{A} \partial_{\boldsymbol{p}}^{B}-\frac{i}{2} \partial_{\boldsymbol{p}}^{A} \partial_{\boldsymbol{R}}^{B}\right) A(\boldsymbol{p}, \boldsymbol{R}) B(\boldsymbol{p}, \boldsymbol{R}) .
$$

Subtracting Eq. (S1) from its conjugate, we obtain

$$
\begin{aligned}
\frac{\boldsymbol{p}}{m_{N}} \nabla \hat{G}+\frac{\alpha}{2}\{\boldsymbol{\eta}, \nabla \hat{G}\}= & {\left[\hat{G}, \omega_{n} \tau_{3}+i \boldsymbol{h}^{\prime} \cdot \sigma^{\prime} \tau_{3}+i h_{z} \sigma_{3} \tau_{3}+i \alpha p \boldsymbol{\eta} \cdot \boldsymbol{n}_{F}+\int d|\boldsymbol{p}| \frac{\langle\hat{G}\rangle}{2 i \tau n \pi}\right] } \\
& +\left\{\partial_{\boldsymbol{R}}(\mu+\Delta+\hat{\Sigma}), \partial_{\boldsymbol{p}} \hat{G}\right\}-\left\{\partial_{p} \hat{\Sigma}, \partial_{\boldsymbol{R}} \hat{G}\right\},
\end{aligned}
$$

where $n=2 \pi p_{F} / v_{F}$ is the density of states times the unit-cell volume and $\langle\cdot\rangle$ means an angular average over the momentum direction. The third term on the right-hand side of Eq. (S6) vanishes because $\partial_{p} \hat{\Sigma}=0$. The second term on the right-hand side of Eq. (S6) is on the order of $\partial_{\boldsymbol{r}}(\mu+\Delta+\hat{\Sigma}) / \boldsymbol{p}_{F}$, which can be neglected. Thus, what is left is

$$
\frac{\boldsymbol{p}}{m_{N}} \nabla \hat{G}+\frac{\alpha}{2}\{\boldsymbol{\eta}, \nabla \hat{G}\}=\left[\hat{G}, \omega_{n} \tau_{3}+i \boldsymbol{h}^{\prime} \cdot \sigma^{\prime} \tau_{3}+i h_{z} \sigma_{3} \tau_{3}+i \alpha p \boldsymbol{\eta} \cdot \boldsymbol{n}_{F}+\int d|\boldsymbol{p}| \frac{\langle\hat{G}\rangle}{2 i \tau n \pi}\right] .
$$


We define the quasiclassical Green's function,

$$
\hat{g}\left(\boldsymbol{R}, \boldsymbol{n}_{F}\right)=\int \frac{d \epsilon_{p}}{i \pi} \hat{G}(\boldsymbol{R}, \boldsymbol{p})=\int \frac{d|\boldsymbol{p}|}{i \pi} \frac{\hat{G}(\boldsymbol{R}, \boldsymbol{p})}{n},
$$

where $\epsilon_{p}$ is the energy dispersion of the lower band in the normal-state $\epsilon_{p}=p^{2} / 2 m-\sqrt{\alpha^{2} p^{2}+h_{z}^{2}}$. Thus, the disorder selfenergy can be written in terms of the quasiclassical Green's function,

$$
\int d|\boldsymbol{p}| \frac{\langle\hat{G}\rangle}{2 i \tau n \pi}=\frac{\langle\hat{g}\rangle}{2 \tau} \text {. }
$$

In this system, the upper band is far away from the Fermi level such that we can project Eq. (S7) onto the lower band. As the lower band has a well-defined Fermi surface, the Green's function has a sharp peak at $\boldsymbol{p}=\boldsymbol{p}_{F}$. Thus, we can replace $\boldsymbol{p}$ by $\boldsymbol{p}_{F}$ in Eq. (S7). This results in

$$
\boldsymbol{v}_{F} \hat{\boldsymbol{\nabla}} \hat{g}=\left[\hat{g}, \omega_{n} \tilde{\tau}_{3}+i \frac{\alpha p_{F}}{S_{F}}\left(\boldsymbol{h}^{\prime} \times \hat{z}\right) \cdot \boldsymbol{n}_{F}+\langle\hat{g}\rangle / 2 \tau\right] .
$$

In this method, the system becomes an effective one-band system after the projection. So, it is natural to replace $\boldsymbol{p}$ by $\boldsymbol{p}_{F}$ outside the Green's function. However, in the paper, we perform it in a different order. We first replace $\boldsymbol{p}$ by $\boldsymbol{p}_{F}$ and then perform the projection. This is also correct. Before the projection, Eq. (S7) has information of both bands, but the contribution of the upper band is very small. When we replace $\boldsymbol{p}$ by $\boldsymbol{p}_{F}$, this only generates a negligible error for the upper band, which does not affect the results.

This work is supported by the Academy of Finland project HYNEQ (Project No. 317118). 